PramaNA
- journal of

Vol. 52, No. 6

June 1999

physics

pp. $537-552$

\title{
A local view of the Kondo effect: Scanning tunneling spectroscopy
}

\author{
WOLF-DIETER SCHNEIDER \\ Institut de Physique de la Matière Condensée, Université de Lausanne, CH-1015 Lausanne, \\ Switzerland \\ E-mail: wschneid@ipmc.unil.ch
}

MS received 4 May 1999

\begin{abstract}
The fascinating many-body physics involved in the interaction of a single magnetic impurity with the conduction electrons of its nonmagnetic metallic host is reflected in unconventional phenomena in magnetism, transport properties and the specific heat. Characteristic low-energy excitations, termed the Kondo resonance, are generally believed to be responsible for this striking behaviour. However, in spite of an intense research for over more than 30 years, a direct spectroscopic observation of the Kondo resonance on individual magnetic adatoms withstood experimental efforts hitherto. The development of low-temperature scanning tunneling microscopes (STM) operating under ultrahigh vacuum (UHV) conditions has provided new opportunities for investigating locally the electronic structure at surfaces. At low temperatures, due to the reduced broadening of the Fermi level of the STM tip and the sample, rather high energy resolution $(\leq 1 \mathrm{meV})$ in scanning tunneling spectroscopy (STS) is achievable. Moreover, the absence of diffusion together with the spatial resolution of the STM enables detailed studies of electronic states on and near single adsorbed atoms and other nanoscale structures. Recently, for the first time, two such STS/STM experiments spatially resolved the electronic properties of individual magnetic adatoms displaying the Kondo effect. In particular, the observed Fano lineshape of the Kondo resonance reveals unambiguously the details of the quantum mechanical interference between the localized orbital and the conduction electrons on an atomic length scale $[1,2]$. This achievement of the detection of individual magnetic atoms with atomic resolution opens new perspectives for probing magnetic nanostructures.
\end{abstract}

Keywords. Kondo effect; Kondo resonance; Fano resonance; individual magnetic atoms; magnetic nanostructures; scanning tunneling spectroscopy; scanning tunneling microscopy.

PACS Nos $72.10 ; 72.15 ; 75.20 ; 71.27 ; 61.16 ; 73.20$

\section{Introduction}

The physics underlying the scattering of conduction electrons by an impurity atom in a metallic host has been first elucidated by Friedel [3]. The perturbing atom induces a change in the electron density of states resulting in a charge oscillation (Friedel oscillation) around the impurity atom. This modification of the density of states (DOS) is of particular importance in the case of a resonant conduction electron - impurity scattering associated with an unfilled $d$ or $f$ level involving the interaction of the electron spin of the impurity with the spin of the conduction electrons: the Kondo effect. The scattering of conduction electrons 
from transition or rare earth metal impurities in nonmagnetic hosts has aroused considerable interest since Kondo's discovery of a low-temperature logarithmic divergence in the scattering amplitude [4]. The most natural description of a single magnetic atom embedded in a nonmagnetic metallic host is provided by the Anderson single impurity model (SIM) [5] which treats localized and extended states on an equal footing. In the formulation of Gunnarsson and Schönhammer [6] the SIM successfully predicted excitation spectra for electron removal (e.g. photoemission) and electron addition (e.g. inverse photoemission) spectra [7, 8]. The essential ingredients of this model are the conduction band of the metallic host in which the localized $d$ or $f$ level of the magnetic impurity is immersed at an energy $\epsilon$ below the Fermi energy $E_{\mathrm{F}}$. Addition of a further electron to the localized state orbital costs the Coulomb interaction (correlation) energy $U$ yielding a total energy of the two electron system of $\epsilon+U$. Consequently the existence of a magnetic moment, i.e., of an unpaired electron spin, requires $\epsilon$ to be below $E_{\mathrm{F}}$ (occupied state) and $\epsilon+U$ above $E_{\mathrm{F}}$ (empty state). The coupling or hybridization between the localized state at $\epsilon$ and the continuum states not only leads to a broadening of the localized state but also to a continuous admixture of $d$ or $f$ symmetry states to the conduction band states of the host in the whole energy range spanned by $U$ including the continuum states around $E_{\mathrm{F}}$. The picture that has emerged to explain the observed unconventional phenomena in magnetism, transport properties and the specific heat is that the local moment is gradually screened by the conduction electrons of the metal host as the temperature approaches $T=0 \mathrm{~K}$ and a many-body non-magnetic singlet ground state is formed around the Fermi energy. These low-energy excitations with a characteristic width of $\delta=k T_{\mathrm{K}}\left(T_{\mathrm{K}}\right.$ is the Kondo temperature and $k$ the Boltzmann constant), known as Abrikosov-Suhl [9, 10] or Kondoresonance [4], contain all the pertinent information on the quantum physics involved in correlated electron systems [5, 6, 11-13] (figure 1). However, the measurement of the unique properties of the Kondo resonance created by the interaction of a single magnetic atom with a nonmagnetic metallic host, its predicted temperature dependence leading to its collapse for temperatures above $T_{\mathrm{K}}$, its predicted splitting in an external magnetic field [13], and the spatial extension of the Kondo screening cloud of the conduction electrons [15] still constitutes a major challenge in condensed matter physics.

Spectroscopic information on the existence and on the temperature dependence of the Kondo resonance in a number of homogeneous dense Kondo or heavy fermion compounds containing rare earth elements with their highly localized $4 f$ electrons has been obtained by high-resolution photoelectron spectroscopy [16-20] and inverse photoemission [21,22]. In general, the overall shape, width and the temperature dependence of the measured spectral function, i.e., of the Kondo resonance at the Fermi energy and of the higher-lying atomic-like excitations reflecting the multiplet structure of the localized states, have been successfully described within the Anderson single impurity model (SIM) [16, 12, 23] (see figure 1). However, electron spectroscopies average over a typical surface area of $1 \mathrm{~mm}^{2}$ with an information depth of a few atomic layers (due to the short electron mean free path inside the solid at typical kinetic energies between $50 \mathrm{eV}$ and $200 \mathrm{eV}$ ). In addition, in spite of the relatively high photoionization cross section for electrons in high orbital momentum states, at impurity concentrations below a few atomic per cent, the spectroscopic signature of a single magnetic impurity embedded in a non-magnetic host remains elusive.

This drawback exists also for the usual measurements on alloys with homogeneously distributed magnetic impurities, such as the temperature dependence of the electrical conductivity or the specific heat which involve various types of averages over the scattering 


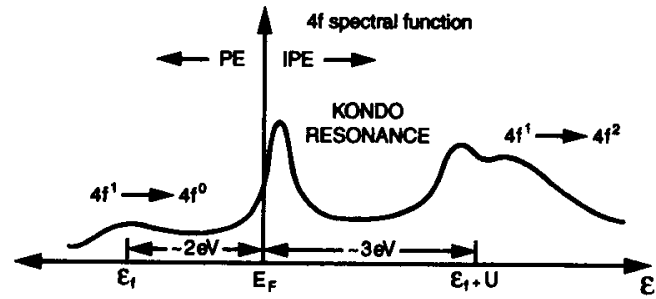

Figure 1. Schematic representation of the $4 f$ spectral function of a single magnetic impurity $(\mathrm{Ce})$ in a conduction electron gas within the Kondo picture. The region below (above) the Fermi energy $E_{\mathrm{F}}$ corresponds to the removal (addition) of one $f$ electron and can be studied by photoemission, PE (inverse photoemission, IPE). After refs [12, $14,18]$.

amplitude but do not measure the scattering amplitude itself. Mezei and Zawadowski [15] suggested, by taking into account the momentum dependence of the exchange integral, that a metal-insulator-metal tunnel (MIM) junction with a layer-like distribution of magnetic impurities situated in one of the metal electrodes close to the electrode-insulator interface, should display a peak in the dynamic resistance versus bias voltage ( $\mathrm{d} V / \mathrm{d} I$ vs $V$ ) around zero bias, directly proportional to the non-spin-flip scattering amplitude. Following this proposal Bermon and So [24] placed a submonolayer of magnetic impurities at different distances of 1 to $10 \mathrm{~nm}$ from the metal-oxide interface into the metal electrode of a MIM tunnel junction condensed on a liquid-helium-cooled substrate. They observed narrow peaks about zero bias in the dynamic tunneling resistance from which the energy dependence of the scattering amplitude in the Kondo effect and the momentum dependence of the $s$ - $d$ exchange integral were deduced. In particular, the decrease in the amplitude of the $\mathrm{dV} / \mathrm{d} I$-peak with the distance of the magnetic impurity layer from the interface agrees with the predictions of ref. [15]. This spatial dependence is characterized by a coherence length $\xi_{\Delta}$, given by $\xi_{\Delta}=\hbar v_{\mathrm{F}}$, where $v_{\mathrm{F}}$ is the Fermi velocity, and $\Delta$ is the energy width over which the exchange integral $J$ changes essentially. For the dilute alloys $\mathrm{Au}-\mathrm{Cr}, \mathrm{Au}-$ $\mathrm{Fe}, \mathrm{Ag}-\mathrm{Fe}$, and $\mathrm{Au}-\mathrm{Mn}$ the authors [24] obtain typical coherence lengths of $\xi_{\Delta}=3.5 \mathrm{~nm}$ and values for $\Delta$ of $0.23 \mathrm{eV}$ for the characteristic energy dependence of $J$. This was the first experiment which provided a direct measure of the Kondo screening length under controlled experimental conditions. Tunneling experiments through point-contact tunnel barriers detected also features in the spectra which have been attributed to the Kondo resonance [25-27]. Subsequent experiments with metal films and wires [28,29] yield different estimates of the spatial extent of the Kondo screening cloud, but the problem inherent to all these measurements is that they are performed on systems which do not represent a single, well-characterized magnetic impurity in a nonmagnetic host.

An important step down in scale has been achieved recently: transport measurements as a function of temperature and magnetic field revealed salient features of the Kondo resonance in metallic quantum dots containing only about 50 atoms [30, 31]. Theory predicts [32-36] that a Kondo singlet should form in a single-electron transistor (SET), which contains a confined droplet of electrons coupled by quantum-mechanical tunneling to the delocalized electrons in the transistor's leads. In such a SET the number of electrons can be changed deliberately from odd to even, the difference in energy between the localized state in the droplet and the Fermi level of the leads can be tuned, the coupling to the leads can be 
adjusted, voltage differences can be applied to reveal non-equilibrium Kondo phenomena [35], and a single localized state can be studied rather than a statistical distribution. The recent measurements of Goldhaber-Gordon et al [30] and of Cronenwett $e t$ al [31] on these small quantum dots reveal all the predicted features of the Kondo resonance. In particular, a tunable Kondo effect has been realized where the dot can be switched from a Kondo system to a non-Kondo system as the number of electrons in the dot is changed from odd to even. The Kondo temperature is tuned by means of the gate voltage as a single-particle energy state approaches the Fermi energy. Measurements of the temperature and magnetic field dependence of the Kondo resonance show good agreement with the predictions for both, the equilibrium and nonequilibrium Kondo effect.

However, in spite of intense research efforts for over more than 30 years, a direct spectroscopic observation of the Kondo resonance on individual magnetic adatoms has not been obtained until recently. The development of low-temperature scanning tunneling microscopes (STM) operating under ultrahigh vacuum (UHV) conditions has provided new opportunities for investigating locally the electronic structure at surfaces. At low temperatures, due to the reduced broadening of the Fermi level of the STM tip and the sample, rather high energy resolution $(\leq 1 \mathrm{meV})$ in scanning tunneling spectroscopy (STS) is achievable. Moreover the absence of diffusion together with the spatial resolution of the STM enables detailed studies of electronic states on and near single adsorbed atoms and other nanoscale structures. For example, STS has been used to perform local spectroscopy in a study of Fe atoms on a clean Pt(111) surface [37]. Resonances of $0.5 \mathrm{eV}$ width were found in the adatom local density of states (LDOS) above the Fermi level. Characteristic circular standing wave patterns around single adatoms have been

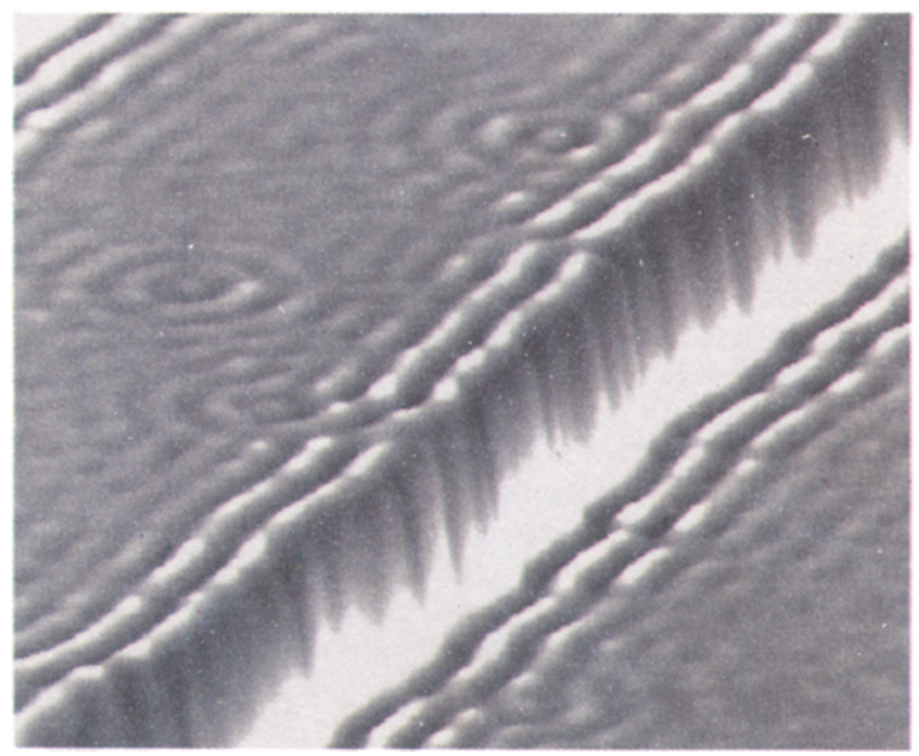

Figure 2. Spectroscopic image $(\mathrm{d} I / \mathrm{d} V)$ of electron standing waves near a monatomic step on $\mathrm{Ag}(111)$ at $5 \mathrm{~K}$. The circular waves are due to electron scattering from impurities (tunneling parameters $V=20 \mathrm{mV}, I=2 \mathrm{nA}$, area $=20 \mathrm{~nm} \times 20 \mathrm{~nm}$ ). After ref. [43]. 
detected in spatially resolved spectroscopic STM images [38-40], providing a breathtaking visualization of quantum mechanics: energy-resolved Friedel oscillations [3] of surface state electrons (figure 2). Recently, the local pair-breaking effect of a single magnetic adatom on a classical superconductor has been observed with STS [41]. In this review we report on the effects of magnetic $\mathrm{Ce}$ and Co adatoms on the LDOS of $\mathrm{Ag}(111)$ and $\mathrm{Au}(111)$, respectively. For the first time two recent STS/STM experiments spatially resolve on an atomic length scale, the electronic properties of individual magnetic adatoms displaying the Kondo effect $[1,2]$.

\section{Spectroscopic evidence of the Kondo resonance of an individual magnetic atom}

\subsection{Cerium on silver [1]}

Experiments with magnetic Ce adatoms were performed with a home-built ultrahigh vacuum (UHV) low-temperature STM [42]. The $\mathrm{Ag}$ (111) surface was prepared by Ar ion bombardment and annealing cycles. Isolated $\mathrm{Ce}$ atoms and, for comparison, isolated $\mathrm{Ag}$ atoms on $\mathrm{Ag}(111)$ were deposited by evaporation from a tungsten filament onto the $\mathrm{Ag}$ substrate at $T=5 \mathrm{~K}$. Subsequently the surface was imaged to detect the isolated adatoms on the $\mathrm{Ag}(111)$ terraces. In figure 3 and figure $4 \mathrm{a}$ the adatoms appear as protrusions with $\approx 0.9 \AA$ and $\approx 1.2 \AA$ height for $\mathrm{Ag}$ and $\mathrm{Ce}$, respectively, with typical widths of $\approx 15 \AA$ in constant current topographs taken at a tunneling resistance of a few hundred $M \Omega$ [43]. For tunneling spectroscopy at the site of the isolated adatoms on the $\mathrm{Ag}(111)$ surface, the tunneling current $I$ and the differential conductance $\mathrm{d} I / \mathrm{d} V$ versus the sample bias voltage $V$, which in simple models is related to the LDOS above the surface $[44,45]$, are recorded while the STM feedback loop is opened. A voltage modulation $\left(243 \mathrm{~Hz}, V_{m}=2-4 \mathrm{mV} \mathrm{rms}_{\text {s }}\right.$ )

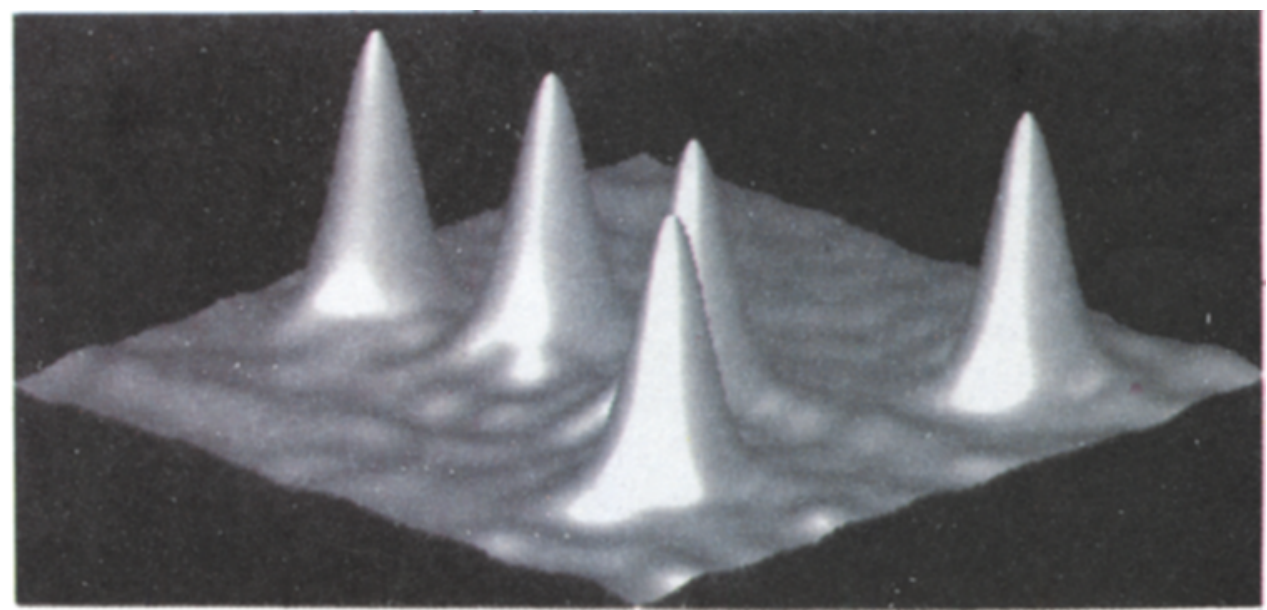

Figure 3. Pseudo three-dimensional constant-current image (low-pass filtered) of $5 \mathrm{Ag}$ adatoms on $\mathrm{Ag}(111)$ at a temperature of $T=5 \mathrm{~K}$ (tunneling parameters $V=200 \mathrm{mV}$, $I=1 \mathrm{nA}$, arca $=6.5 \mathrm{~nm} \times 6.5 \mathrm{~nm})$. After ref. [43]. 

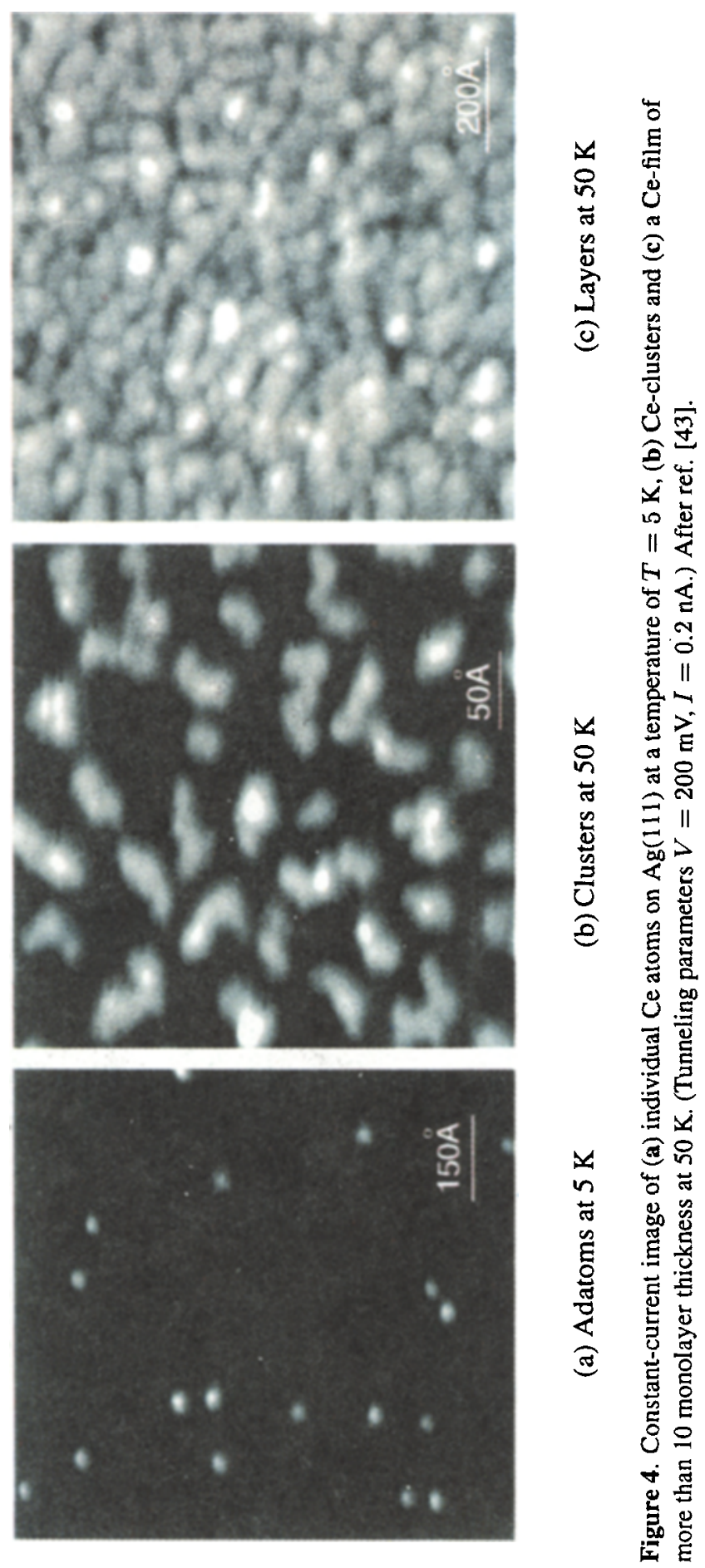
is added to $V$ and a lock-in amplifier is used to measure $\mathrm{d} I / \mathrm{d} V$. These tunneling spectra were measured on several Ce adatoms using three electrochemically etched $W$ tips which were prepared in UHV by heating and Ar ion bombardment. Each of these tips was repeatedly and intentionally modified by applying voltage pulses and by bringing it into contact with the Ag surface. The spectroscopic features discussed below were observed with all these tips over a range of tip-sample distances defined by the tunneling parameters $(500$ $\mathrm{mV}>V>-500 \mathrm{mV}, 100 \mathrm{pA}<I<2 \mathrm{nA}$, corresponding to tunneling resistances in the range $M \Omega$ to $G \Omega$ ) used prior to opening the feedback loop.

Ce impurity atoms have been chosen because of their known magnetic behavior in bulk Ce compounds [19] and their high $T_{\mathrm{K}}(\approx 1000 \mathrm{~K}$ in $\alpha$-Ce [12, 20]). Figure 5b displays a $\mathrm{d} I / \mathrm{d} V$ tunneling spectrum measured on top of an isolated Ce adatom (figure 4a). A clear depression or antiresonance is observed in $\mathrm{d} I / \mathrm{d} V$ around the Fermi energy. When the tip is moved laterally away from the impurity atom this antiresonance vanishes already at a lateral distance of $5 \AA$ from the center of the magnetic adatom and on the $\mathrm{Ag}(111)$ substrate the typical spectrum of the $\mathrm{Ag}(111)$ surface state is observed (figure 5e), with the surface state band edge at $E=-70 \mathrm{meV}$ and a surface band at higher energies [46, 47]. At intermediate distances (figure 5c,d) broadening of the surface state edge is observed reffecting a decreased hot-hole lifetime due to scattering of surface state electrons at the impurity $[48,49]$. The weak spectral features above the onset of the surface state are due to standing wave patterns caused by scattering at the adatoms $[38,39,46]$. These energy-resolved Friedel oscillations are also visible in space in the constant current image of figure 3 and in the spectroscopic image of figure 2 as weak circular waves around the adatoms. We repeated the same kind of measurements on Ce clusters (figure $4 \mathrm{~b}$ ) and on thin Ce films at $T=50 \mathrm{~K}$ (figure $4 \mathrm{c}$ ). As shown in figure 6 the characteristic dip around

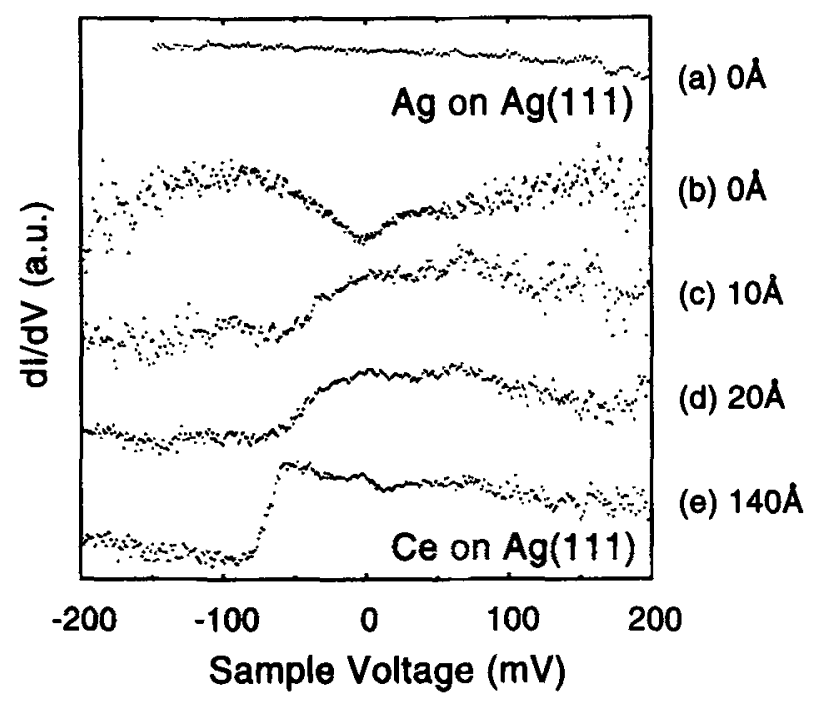

Figure 5. $\mathrm{d} I / \mathrm{d} V$ spectra on (a) a single $\mathrm{Ag}$ adatom and (b)-(e) on and near a single $\mathrm{Ce}$ adatom at $T=5 \mathrm{~K}$. The lateral distance between the tip position and the center of the adatom is indicated. Before opening the feedback loop the tunnel parameters were $V=200 \mathrm{mV}, I=0.1 \mathrm{nA}$. After ref. [1]. 


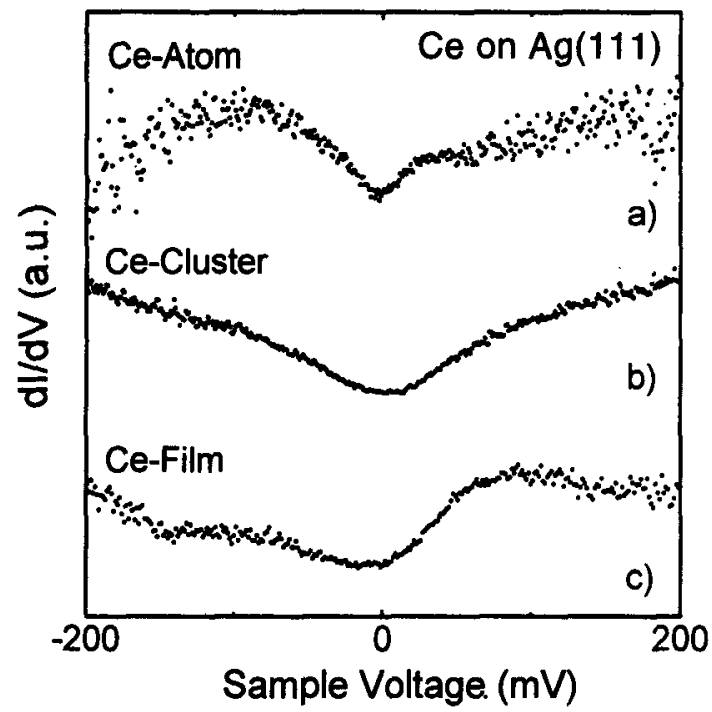

Figure 6. $\mathrm{d} I / \mathrm{d} V$ spectra (a) on a single Ce adatom at $T=5 \mathrm{~K}$, (b) on a Ce-cluster at $T=50 \mathrm{~K}$, (c) on a Ce-film of more than 10 monolayer thickness at $T=50 \mathrm{~K}$. Tunneling parameters prior to opening the feedback: (a) $V=200 \mathrm{mV}, I=0.1 \mathrm{nA}$, (b) $V=500 \mathrm{mV}, I=1 \mathrm{nA}$, (c) $V=350 \mathrm{mV}, I=1 \mathrm{nA}$. After ref. [1].

the Fermi level is always observed. Its width, however, increases from about $50 \mathrm{mV}$ on top of the Ce adatom to about $100 \mathrm{mV}$ on a Ce film. For comparison, tunneling spectra of a single adsorbed $\mathrm{Ag}$ atom serving as a prototype for a nonmagnetic atom were taken. The spectrum taken on top of a $\mathrm{Ag}$ adatom (figure 5a) is featureless throughout the range from $-200 \mathrm{mV}$ to $200 \mathrm{mV}$. Such a situation has been observed previously at monatomic steps and is attributed to quenching of the $\mathrm{Ag}(111)$ surface state [46]. The comparison between figures $5 \mathrm{a}$ and $\mathrm{b}$ clearly indicates that the dip or antiresonance near the Fermi energx $E_{\mathrm{F}}$ is indeed characteristic of the magnetic $\mathrm{Ce}$ adatom. This observation appears to contradict recent theoretical investigations [50] which, similar to figure 1, predict a sharp Kondo resonance in $\mathrm{d} I / \mathrm{d} V$ spectra near the Fermi energy.

To interpret these spectroscopic observations model calculations within the Anderson SIM for a single magnetic $4 f$ impurity adsorbed on a nonmagnetic metallic host were performed using the zero temperature Gunnarsson-Schönhammer theory [6] and the slave boson approach in the non-crossing approximation (NCA) [23, 17]. For reasonable parameters $(k T=1 \mathrm{meV}$, bare $4 f$ energy $(-1.2 \mathrm{eV}), 4 f$ spin orbit splitting $(280 \mathrm{meV})$, crystal field splitting neglected and the $4 f$-conduction band hybridization $(60 \mathrm{meV})$ in the range of published values for CeAg alloys [51]) Kondo peaks appear on an energy scale which matches that of the observed antiresonance.

A closer inspection of the tunneling process led the authors [1] to an explanation for the existence of an antiresonance in the tunneling spectra as opposed to the resonance predicted by theory [50] and measured by photoemission for similar systems. The probing of a localized state immersed in a continuum by the tunneling microscope resembles the spectroscopy of a discrete autoionized state, which has been elucidated by Fano [53]. An electron tunneling from the STM tip to, e.g., the empty states of the Kondo resonance above 


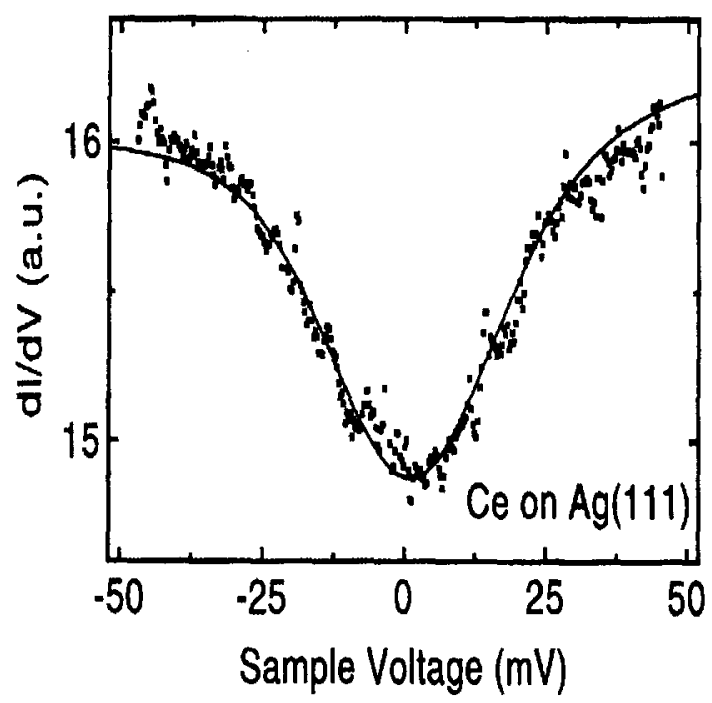

Figure 7. $\mathrm{d} I / \mathrm{d} V$ spectra of a Ce impurity on $\mathrm{Ag}(111)$ for an energy range around the Fermi level. Squares: measurement $(V=100 \mathrm{mV}, I=0.1 \mathrm{nA}$ before opening the feedback loop). Solid line: Calculation using the Anderson single impurity model (see text). After ref. [1].

$E_{\mathrm{F}}$ (sample voltage $V \geq 0$ ) can occupy two energetically degenerate possible final states: the modified (hybridized) localized state and the continuum of band states. This situation leads to quantum interference. In the limit where the transition matrix element for the localized state approaches zero ( $q \approx 0$ case in ref. [53]), the autoionization spectrum shows a dip or antiresonance, as a consequence of the interference effects at the site with the localized state. The width of the antiresonance is on the same scale as that of the resonance. Indeed, at large tip to surface distances, the STM is known to probe $s p$ wave functions rather than the confined $4 f$-derived Kondo ground state [52]. Thus the $\mathrm{d} I / \mathrm{d} V$ spectrum should show an antiresonance instead of a $f$-spectral peak as observed experimentally. By approximating the Fano dip shape by the inverse NCA resonance [1] the lineshape in figure 7 (solid curve) is obtained, which is in encouraging agreement with the experimental observations (dots). The small asymmetry observed in the experimental spectrum with respect to the Fermi energy is interpreted as a manifestation of the Fano-interference effect which occurs when there is a small transition pı ubability to/from $f$ symmetry states. The width of the Kondo antiresonance obtained from the NCA calculation for the single isolated Ce impurity on $\mathrm{Ag}(111)$ (figure 7 (solid curve)) is $\delta=k T_{\mathrm{K}}=25(5) \mathrm{meV}$ yielding a Kondo temperature of $T_{\mathrm{K}}=290(50) \mathrm{K}$ which is considerably lower than the value of $1000 \mathrm{~K}$ found for bulk $\alpha$-Ce $[12,20]$. This value is consistent with the observed decrease of the width of the antiresonance in going from the $\mathrm{Ce}$ film to the $\mathrm{Ce}$ cluster and to the Ce adatom reflecting the decrease in hybridization due to reduced overlap between neighboring Ce-atoms.

This finding adds new knowledge to the physics of probing correlated electrons and allows us to treat the spectral functions obtained by photoemission and tunneling spectroscopies on an equal footing. In photoemission, the high $4 f$ photoionization cross section (at photon energies above $30 \mathrm{eV}$ ) with respect to $s p$ band electrons is equivalent to a large Fano parameter $q \approx \infty$. Consequently, essentially only the $4 f$-derived spectral function 
including the low energy excitations, i.e. the Kondo resonance is observed (see figure 1). In STS, the weak overlap of the electronic states of the tip with the $4 f$-wave function of the magnetic impurity is equivalent to $q \approx 0$ and consequently predominantly the $s p$-wave functions are probed. In other words, the Kondo resonance known from photoemission appears as a Kondo antiresonance in the tunneling spectra due to a Fano interference effect. This interpretation has recently been confirmed by similar observations from Co atoms on $\mathrm{Au}(111)$ which also give rise to a typical Fano-lineshape [2] (see next section).

\subsection{Cobalt on gold [2]}

Only shortly afterwards Madhavan et al [2] reported on similar low-temperature STMobservations from $\mathrm{Co}$ atoms on $\mathrm{Au}(111)$ and confirmed the interpretation given by $\mathrm{Li}$ et al [1]. These authors have chosen the $3 d$-metal Co atoms which in bulk Au have Kondo temperatures above $300 \mathrm{~K}[11,54,55]$ and which on Au surfaces are magnetic with $T_{\mathrm{K}}=19 \mathrm{~K}$

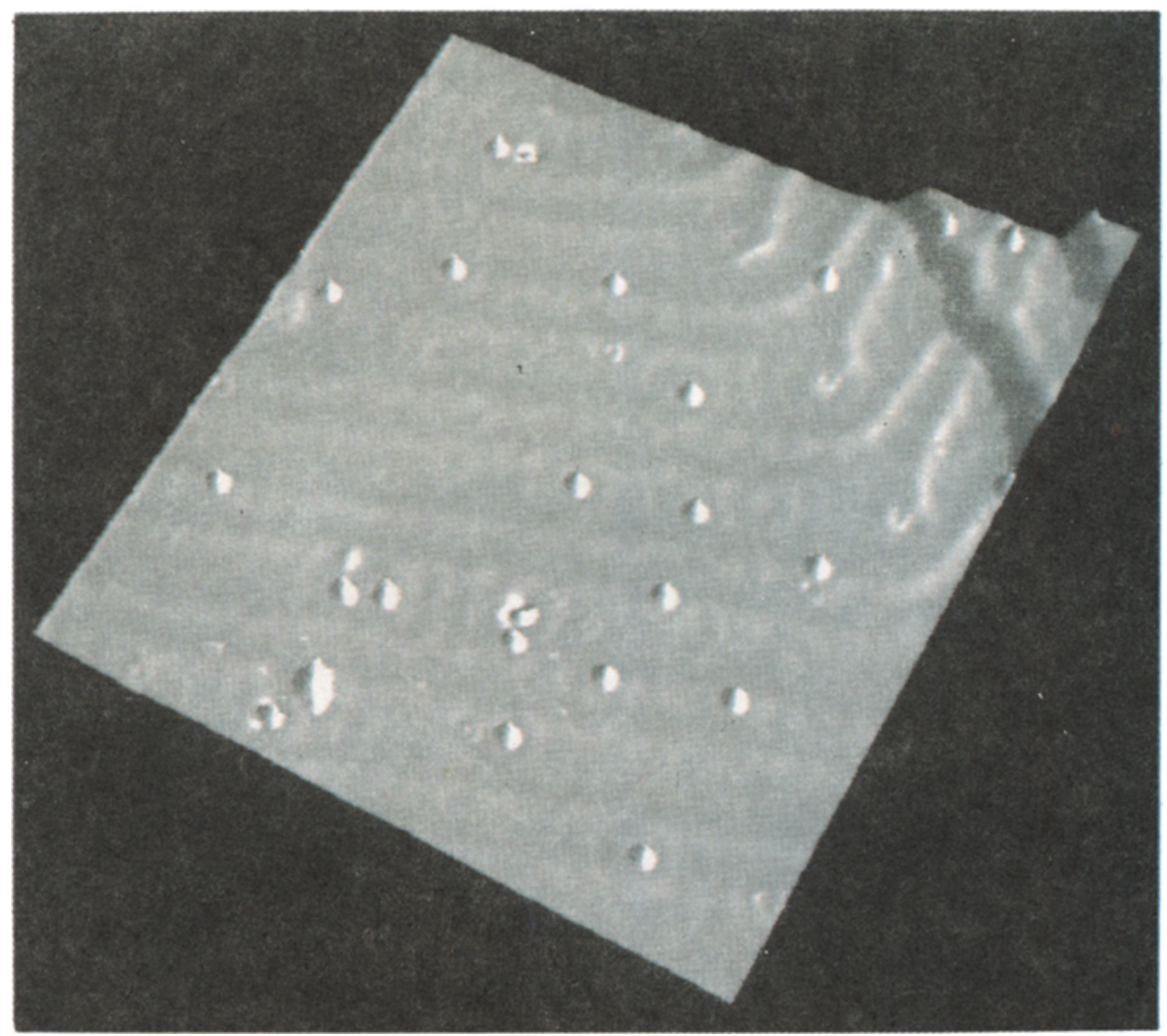

Figure 8. Constant-current image of about 22 individual Co atoms on the reconstructed (herringbone) $\mathrm{Au}(111)$ surface at a temperature of $T=4 \mathrm{~K}$. (tunneling parameters $V=100 \mathrm{mV}, I=0.5 \mathrm{nA}$ ). After ref. [2]. 


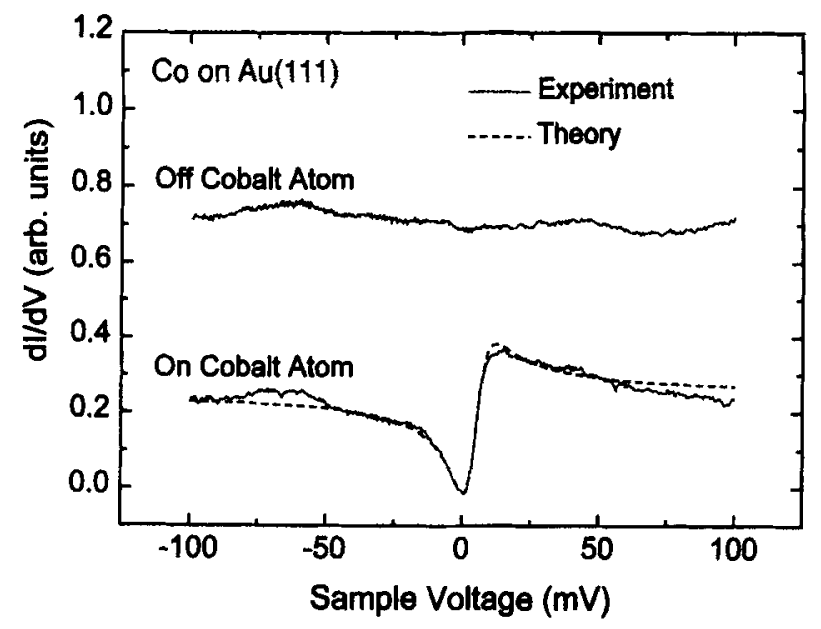

Figure 9. $\mathrm{d} I / \mathrm{d} V$ spectra taken at $T=4 \mathrm{~K}$ on a single Co adatom (bottom) and over the bare $A u(111)$ surface at a lateral distance of $12 \AA$ from the center of the Co adatom (top). The dashed curve shows a fit to the data using a modified Fano theory. After ref. [2].

[56]. The measurements were performed in a homemade STM operated at $4 \mathrm{~K}$ in ultrahigh vacuum with well prepared tips and a clean single crystal Au(111) surface. Co atoms were deposited onto the cold surface using a calibrated evaporator. The bias voltage $V$ across the tunnel junction was defined as the sample voltage with repect to the STM-tip. Figure 8 shows a $40 \times 40 \mathrm{~nm}^{2}$ constant current image of the reconstructed Au(111) surface [57] covered with about 22 Co atoms of $0.8 \AA$ apparent height. Also visible are low-amplitude waves arising from the quantum interference of two-dimensional surface-state electrons on $\mathrm{Au}(111)$ [58] as well as circular waves (Friedel oscillations) around the Co-atoms as observed above for $\mathrm{Ce}$ and nonmagnetic atoms and defects $[38,39,46]$. Scanning tunneling spectroscopy (STS) enabled the authors to measure the spatial variation of the LDOS as a function of the lateral tip distance from the Co atom (as in the Ce case above). The $\mathrm{d} I / \mathrm{d} V$ spectra were obtained with lock-in detection of the ac tunnel current driven by a $450 \mathrm{~Hz}$, $1 \mathrm{mV}$ (root-mean-square) signal added to the junction bias. The tip-surface separation was varied between values corresponding to tunnel junction resistances between $200 \mathrm{M} \Omega$ and $200 \mathrm{k} \Omega$ without that significant changes in the spectra have been observed. These tunnel spectra were taken by opening the STM feedback loop and by subsequently scanning the bias voltage. The authors repeated their measurements on hundreds of different Co atoms with a variety of different tips (by changing the microscopic arrangement of the last few atoms on the STM tip by field emission and by collisions with the surface) and found that the tunnel spectra were very reproducible.

Figure 9 shows the result of these measurements on and off a single Co atom on the Au(111) surface. The $\mathrm{d} I / \mathrm{d} V$ spectrum measured with the STM tip above the center of a single Co atom shows a sharp, asymmetric resonance (bottom solid curve) around $E_{\mathrm{F}}(V=$ 0 ), whereas the spectrum mesured on the $\mathrm{Au}(111)$ surface with the tip moved laterally $12 \AA$ away from the Co atom is nearly featureless (top solid curve). In comparison, spectroscopic measurements of nonmagnetic $\mathrm{Cu}$ atoms on $\mathrm{Au}(111)$ did not show a resonance at $V=0$. Figure 10 shows in finer detail the decrease in amplitude of the resonance and its change 


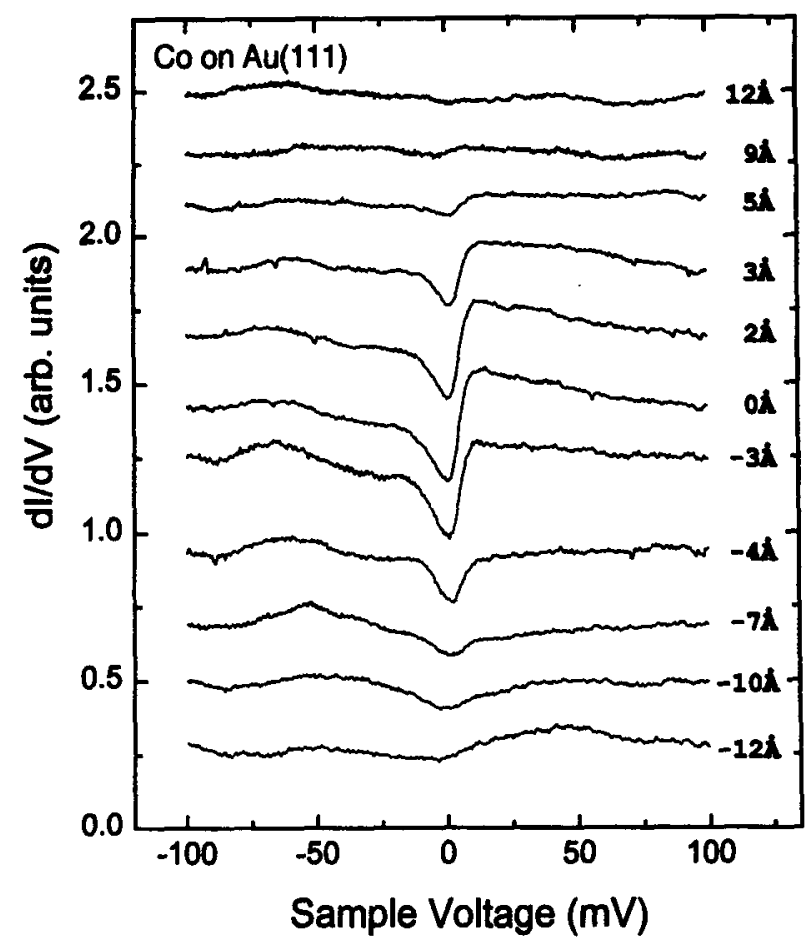

Figure 10. $\mathrm{d} I / \mathrm{d} V$ spectra taken at various lateral spacings from the center of a single Co atom on $\mathrm{Au}(111)$. Data is from the same atom as the data in the preceeding figure. After ref. [2]

towards a more symmetric lineshape when moving the tip laterally away from the center of the Co atom located at $0 \AA$.

Madhavan et al [2] interpreted this characteristic asymmetric lineshape as a manifestation of a Fano interference phenomenon [53] in the tunnel process: tunneling of electrons from the tip to the Co adatom occurs via two channels, into the $d$-orbital of the magnetic impurity and into the surrounding continuum of the conduction-band electrons at the same energy. Fano treated transitions from an initial state to a noninteracting discrete state embedded in a continuum of states. In the Anderson SIM this situation corresponds to a Coulomb correlation energy $U=0 \mathrm{eV}$. Previous theoretical treatment of tunneling into an Anderson impurity [59] enabled the authors to generalize Fano's analysis to an interacting resonant level with $U \neq 0$. The result is that the Fano line shape for the noninteracting case is broadened by $\Delta E=2 k T_{\mathrm{K}}$ in the interacting case. The comparison between this theoretical lineshape (dashed curve in figure 9) with the experiment confirms the validity of this approach. From measurements taken on nine different $\mathrm{Co}$ atoms with different tips average values of the Fano parameter $q=0.6 \pm 0.1$ and of $k T_{\mathrm{K}}=5.8 \pm 0.7 \mathrm{meV}$, corresponding to a Kondo temperature of a Co atom on $\mathrm{Au}(111)$ of about $70 \mathrm{~K}$, have been obtained. This value of $T_{\mathrm{K}}$ is considerably lower than the values known for Co impurities in the bulk $[11,54,55]$, a situation very similar to the one encountered above for $\mathrm{Ce}$ on $\mathrm{Ag}(111)$. The lower $T_{\mathrm{K}}$ at the surface is attributed to the smaller coordination of the impurity atoms at the surface as compared to the bulk leading to reduced overlap of the $d$-impurity orbital 
with conduction-band states and therefore to a narrower Kondo resonance. On the other hand this value of $T_{\mathrm{K}}$ measured on the $\mathrm{Au}(111)$ single crystal surface is larger than the one obtained by a weak-localization technique [56] for Co on a disordered Au film (19 K), a discrepancy attributed to the different surface conditions in both experiments.

The evaluation of the asymmetry of the Fano resonance as a function of the lateral tip position (figure 10) with respect to the Co adatom yields a decrease of the Fano parameter $q$ from $q=0.7$ at the center of the adatom to $q=0.1$ at a distance of $4 \AA$. The authors interpret this finding in terms of a spatially changing transition probability between the tip states and the $d$-orbital channel of the magnetic adatom whereas the transition matrix element to the continuum states stays about constant. The spatial dependence of the Fano profile thus reflects the lateral extension of the Co $d$-orbital.

\section{Conclusions and outlook}

In conclusion, using the tip of a scanning tunneling microscope as a local probe the electronic structure of a prototype of a Kondo-system, a single magnetic adatom on a metallic surface (Ce on $\mathrm{Ag}(111)$ [1] and $\mathrm{Co}$ on $\mathrm{Au}(111)$ [2]) was observed at atomic resolution. In differential conductance $(\mathrm{d} I / \mathrm{d} V)$ spectra the signature of the Kondo effect, the Kondo resonance, was identified at low temperatures $T<T_{\mathrm{K}}$. The characteristic line shape has been interpreted in both cases $(\mathrm{Ce}$ and $\mathrm{Co}$ ) in terms of a Fano interference between transitions to a discrete state and to a continuum of band states in the same energy range. The localization of the $4 f$ and $3 d$ orbitals is reflected in the lineshape of the Fano resonance. A nearly symmetric antiresonance occurs when the tip is placed on top of an individual Ce adatom indicating that the tunneling transition probability to the localized $f$ states is very small $(q \approx 0)$ while the spectrum taken at the center of an individual Co adatom displays a typical asymmetric Fano line shape, indicating an increased tunneling transition probability to the slightly more extended $d$-states $(q=0.7)$. Also the lateral spatial extension of the localized orbitals was found to be larger for $\mathrm{Co}$ than for $\mathrm{Ce}$ in agreement with expectation.

The ability of the STM to detect the electronic structure on the scale of a single magnetic impurity on a surface has not only elucidated the inherent quantum mechanical Fano interference phenomenon but provides us also with the important information of the spatial extent of the Kondo screening cloud of the conduction electrons [15, 24]. The fact that there is no change in the tunnel spectra taken at distances larger than $12 \AA$ away from the center of the $\mathrm{Co}$ adatom and that for $\mathrm{Ce}$ on $\mathrm{Ag}(111)$ the only influence of the impurity seen at these distances is the lifetime change of the surface state would indicate a Kondo screening length $\xi_{\Delta}$ in the range of $10-15 \AA$. This value is about a factor of two smaller than the values found for dilute $\mathrm{Au}-\mathrm{Fe}$ and $\mathrm{Ag}-\mathrm{Fe}$ systems [24]. It is interesting to note that in this short-range region a spatially dependent depletion of the LDOS on and around a magnetic impurity has been predicted by Mezei and Zawadowski [15] while for the longrange region they predict Friedel oscillations as observed experimentally. Moreover, for $s$-type scattering the amplitude of the antiresonane is calculated to take its maximum value at the magnetic impurity site while for $d$-type scattering this maximum value is found at about one atomic distance away from the impurity site [15]. In the light of this calculation the presented tunneling spectra indicate $s$-scattering of the conduction electrons at the magnetic $\mathrm{Ce}$ and $\mathrm{Co}$ impurity.

The present scanning tunneling spectroscopic studies are a first step towards an under- 
standing of magnetic impurity scattering at an atomic level. There is still a lot of work ahead of us. For example, the spectroscopic investigation of a single magnetic impurity as a function of temperature in order to reveal the evolution of the Kondo resonance below and above $T_{\mathrm{K}}$. Moreover, the application of an external magnetic field should split the resonance by the Zeeman energy [11] as has been shown for the quantum dot in the single electron transistor (SET) $[30,31]$. The finding of a change in the width and shape of the Kondo resonance for the Ce-clusters [1] and the observation of the insensitivity of the lineshape to the proximity of other Co atoms for interatom spacings down to $6 \AA$ [2] points to exciting possibilities. The atom manipulation capabilities of the STM [60] should allow us to construct new arrangements of magnetic impurities on a surface and to study their mutual interaction via exchange coupling with the conduction electrons as a function of their interatomic distances. In this way, e.g., a two-dimensional Kondo lattice might be realized. The present achievement of the detection of individual magnetic atoms with atomic resolution opens new perspectives for probing magnetic nanostructures.

\section{Acknowledgements}

The author is pleased to acknowledge a very fruitful and stimulating collaboration with R Berndt, B Delley, J T Li and F Patthey. The author wishes to thank A Zawadowski for an inspiring discussion. This work was supported by the Swiss National Science Foundation.

\section{References}

[1] J T Li, W-D Schneider, R Berndt and B Delley, Phys. Rev. Lett. 80, 2893 (1998)

[2] V Madhavan, W Chen, T Jamneala, M F Crommie and N S Wingreen, Science 280, 569 (1998)

[3] J Friedel, Phil. Mag. Suppl. 3, 446 (1954) J Friedel, Nuovo Cimento Suppl. 7, 287 (1958)

[4] J Kondo, Progr. Theor. Phys. 32, 37 (1964)

[5] P W Anderson, Phys. Rev. 124, 41 (1961)

[6] O Gunnarsson and K Schönhammer, in Handbook on the Physics and Chemistry of Rare Earths edited by K A Gschneidner, L Eyring and S Hüfner (Elsevier Publisher, Amsterdam, 1987) and references therein, vol. 10, pp. 103

[7] Y Baer and W-D Schneider, in Handbook on the Physics and Chemistry of Rare Earths edited by K A Gschneidner, L Eyring and S Hüfner (Elsevier Publisher, Amsterdam, 1987) and references therein, vol. 10, pp. 1-73

[8] J W Allen, in Synchrotron Radiation Research: Advances in Surface and Interface Science edited by R Z Bachrach (Plenum, New York, 1992) vol. 1 pp. 253-323

[9] A A Abrikosov, Physics 2, 5, 61 (1965)

[10] H Suhl, in Theory of Magnetism in Transition Metals edited by W Marshall (Academic, London, 1967) pp. 116-205

[11] G Gruner and A Zawadowski, Rep. Prog. Phys. 37, 1479 (1974)

[12] P A Lee, T M Rice, J W Serene, L S Sham, and J W Wilkins, Comm. Condens. Matter Phys. 12,99 (1986)

[13] A C Hewson, The Kondo Problem to Heavy Fermions (Cambridge Univ. Press, Cambridge, 1993) 
[14] D W Lynch and J H Weaver, in Handbook on the Physics and Chemistry of Rare Earths edited by K A Gschneidner, L Eyring and S Hüfner (Elsevier Publisher, Amsterdam, 1987) and references therein, vol. 10, pp. 231-300

[15] F Mezei and A Zawadowski, Phys. Rev. B3, 167, 3127 (1971)

[16] F Patthey, B Delley, W-D Schneider and Y Baer, Phys. Rev. Lett. 55, 1518 (1985)

[17] F Patthey, W-D Schneider, Y Baer and B Delley, Phys. Rev. Lett. 58, 2810 (1987)

[18] F Patthey, J-M Imer, W-D Schneider, H Beck, Y Baer and B Delley, Phys. Rev. Lett. 42, 8864 (1990)

[19] D Malterre, M Grioni and Y Baer, Adv. Phys. 45, 299 (1996)

[20] M Garnier, K Breuer, D Purdie, M Hengsberger, Y Baer and B Delley, Phys. Rev. Lett. 78, 4127 (1997)

[21] E Wuilloud, H R Moser, W-D Schneider and Y Baer, Phys. Rev. B28, 7354 (1983)

[22] F U Hillebrecht and M Campagna, in Handbook on the Physics and Chemistry of Rare Earths edited by K A Gschneidner, L Eyring and S Hüfner (Elsevier Publisher, Amsterdam, 1987) and references therein, vol. 10, pp. 425-451

[23] P Coleman, Phys. Rev. B29, 3035 (1984)

[24] S Bermon and C K So, Phys. Rev. Lett. 40, 53 (1978)

[25] S Gregory, Phys. Rev. Lett. 68, 2070 (1992)

[26] D C Ralph and R A Buhrman, Phys. Rev. Lett. 72, 3401 (1994)

[27] E L Wolf, Principles of Electron Tunneling Microscopy (Oxford Univ. Press, New York, 1985)

[28] V Chandresakar, P Santhanam, N A Penebre, R A Webb, H Vloeberghs, C Van Haesendonck and Y Bruynseraede, Phys. Rev. Lett. 72, 2053 (1994)

[29] M A Blachly and N Giordano, Phys. Rev. B51, 121537 (1995)

[30] D Goldhaber-Gordon, Hadas Shtrikman, D Mahalu, David Abusch-Magder, U Meirav and M A Kastner, Nature 391, 156 (1998)

[31] S M Cronenwett, Tjerk H Oosterkamp and Leo P Kouwenhoven, Science 281, 540 (1998)

[32] T K Ng and P A Lee, Phys. Rev. Lett. 61, 1768 (1988)

[33] L I Glazman and M E Raikh, JETP Lett. 47, 452 (1988)

[34] Y Meir, N S Wingreen and P A Lee, Phys. Rev. Lett. 70, 2601 (1993)

[35] N S Wingreen and Y Meir, Phys. Rev. B49, 11040 (1994)

[36] R Lopez, R Aguado, G Platero and C Tejedor, Phys. Rev. Lett. 81, 4688 (1998)

[37] M F Crommie, C P Lutz and D M Eigler, Phys. Rev. B48, 2851 (1993)

[38] M F Crommie, C P Lutz and D M Eigler, Nature 363, 524 (1993)

E J Heller, M F Crommie, C P Lutz and D M Eigler, Nature 369, 464 (1994)

[39] Y Hasegawa and Ph Avouris, Phys. Rev. Lett. 71, 1071 (1993)

Ph Avouris and I-W Lyo, Science 264, 942 (1994)

[40] J T Li, W-D Schneider and R Berndt, Phys. Rev. B56, 7656 (1997)

[41] A Yazdani, B A Jones, C P Lutz, M F Crommie and D M Eigler, Science 275, 1767 (1997)

[42] R Gaisch, J K Gimzewski, B Reihl, R R Schlittler, M Tschudy and W-D Schneider, Ultramicroscopy 42-44, 1621 (1992)

[43] J T Li, Manipulation and spectroscopy at the atomic level, $\mathrm{PhD}$ thesis (University of Lausanne, Switzerland, 1997)

[44] J Tersoff and D R Hamann, Phys. Rev. B58, 45 (1987)

[45] N D Lang, Phys. Rev. B34, 5947 (1986)

[46] J T Li, W-D Schneider, R Berndt and S Crampin, Phys. Rev. Lett. 80, 3332 (1998)

[47] R Paniago, R Matzdorf, G Meister and A Goldmann, Surf. Sci. 336, 113 (1995)

[48] J T Li, W-D Schneider, R Berndt, O R Bryant and S Crampin, Phys. Rev. Lett. 81, 4464 (1998)

[49] J T Li, W-D Schneider, S Crampin and R Berndt, Surf. Sci. 422, 95 (1999)

[50] S Hershfield, J H Davies, and J W Wilkins, Phys. Rev. Lett. 67, 3720 (1991)

[51] P Monachesi, L C Andreani, A Continenza and A K McMahan, J. Appl. Phys. 73, 6634 (1993) 
[52] N D Lang, Phys. Rev. Lett. 58, 45 (1987)

[53] U Fano, Phys. Rev. 124, 1866 (1961)

[54] D Riegel and K D Gross, Physica B163, 678 (1990)

[55] M D Daybell and W A Steyert, Rev. Mod. Phys. 40, 380 (1968)

[56] W Wei, R Rosenbaum, and G Bergmann, Phys. Rev. B39, 4568 (1989)

[57] J V Barth, H Brune, G Ertl and R J Behm, Phys. Rev. B42, 9307 (1990)

[58] S D Kevan and R H Gaylord, Phys. Rev. B36, 5809 (1987)

[59] Y Meir and N S Wingreen, Phys. Rev. Lett. 68, 2512 (1992)

[60] D M Eigler and E K Schweizer, Nature 344, 524 (1990) 